\title{
Photothermal inspections of adhesion strengths and detection of delaminations
}

\author{
by R.Ritter and B.Schmitz
}

Institut für Lasertechnologien in der Medizin und MeBtechnik an der Universität UIm, Helmholtzstraße 12, D-89081 UIm, Germany

\begin{abstract}
For more than a decade, photothermal measurement techniques have been used for the nondestructive and contactless evaluation of coating thicknesses and for the investigation of boundaries between a surface layer and its base material. In the following sections, we aim to describe a few examples whereas the photothermal radiometry has been applied on different interfaces embedded in an optically opaque workpiece. The effect of adhesion defects on photothermal signals will be demonstrated. The obstructed propagation of thermal waves can be explained by thermal contact resistances when delaminations or disbondings are located between a coating and its substrate.
\end{abstract}

\section{Introduction}

For several engineering purposes, the coating (lacquer coatings, galvanically deposited layers etc.) has to fulfill well-characterized demands. Certainly, it has to improve the functionality of workpieces. The interfaces between the coating and the substrate should be well characterized and without any faults. One of the main defects found in coated metal substrates is lack of adhesion between surface layer and base material. These delaminations might be caused by poorly prepared base substrates, by defective boundaries between coating and substrate or by varied composition and porosity of the coating material. Altogether, the coating, its adhesion strength or the delaminations and subsurface defects determine decisively the applicability and reliability of plated parts. Both from an engineering and economic point of view, the determination of the coating's thickness and adhesion strength as well as its control during the manufacturing process become increasingly important.

For satisfactory in-line manufacturing control, contactless and non-destructive measurement techniques are demanded. Optical methods yield an overview of the outer surface, only. In order to get more sets of information about the inner structures (kind of inclusion, near-surface properties of the interfacial material causing the delamination), it is necessary to extend optical methods. In order to be applied on coatings they have to determine both the integrity (e.g. the absence of adhesion defects) and the thickness of a given coating on a workpiece. The methods must be applicable to sticky and wet coatings as well as to the extensive range of geometrical configurations found in engineering.

During the last 15 years, photothermal measurement techniques [1] were shown to fulfil some of the aforementioned conditions and, hence, being suitable for a non-destructive evaluation of coating thicknesses [2-4], subsurface buried defects, thermal inhomogeneities and hidden structures [5] or for the characterization of mechanically or thermally induced variations of various properties in near-surface layers [6-8]. Based on the analysis of the propagation and attenuation of optically excited Thermal Waves, photothermal methods allow for depth profiling of thermal properties. Each thermal inhomogeneity, e.g., inclusion, subsurface defect or buried structure, disturbs the propagation of the thermal waves and modifies the temperature response on the sample's surface - compared with the surrounding flawless areas. Due to the immediate correlation between thermal and mechanical parameters, the temperature signals map the mechanical properties. 
For our investigations on the determination of coating thicknesses and the characterization of thermal contact resistances between layer and base materials we chose the photothermal radiometric technique. In order to get some information about the interface layers between coating and base material, we analyse the frequency dependence of the photothermal phase signal very accurately. In contrast to the magnitude of the signal, the phase is mainly independent on the optical properties and assumes only surface absorption.

\section{Theoretical background of thermal waves propagation at an interface}

Usually, interfaces are characterized by a more or less abrupt change of the material properties. One assumes that these mechanical alterations (e.g. at interfaces, grain boundaries, microstructural pattern or coating-substrate boundaries) are correlated with varied thermal properties and, therefore, become detectable by photothermal means. So far, figure 1 illustrates the theoretical basis for the photothermal inspection of layer thickness and thermal contact resistances. While a sharp thermal transition (curve 1 in figure 1 ) is assumed for an unaffected boundary between coating and substrate (in particular, this assumption yields the photothermal determination of a layer's thickness) a delamination or defected adhesion strength is theoretically assumed by a thermal contact resistance (curve 2 in figure 1). The heat transfer between the two layers is disturbed and the surface temperature is modified by the thin transition zone. The increased reflectivity for the thermal wave causes an enhanced temperature response on the surface and a phase shift of the reflected thermal wave. Thus, an accurate analysis of the frequency dependence of the photothermal signals yields the characterization of subsurface features.

Compared with the single thermal reflection coefficient at a steplike transition (figure 1 , line 1) two important changes must be realized:

- The reflection coefficient of a defective boundary must be described by complex functions and, therefore, the reflected wave is phase shifted.

- The reflection coefficient of a defective boundary layer depends on the modulation frequency. Thus, the detectability of a disbonding or a delamination depends on the modulation frequency, too.

\section{Experimental setup}

Several photothermal measurements have been performed with a photothermal radiometric microscope as sketched in figure 2. The thermal waves were generated by an argon ion laser whose intensity can be modulated by an acousto-optical device. A part of the heat radiation emitted from the surface of the sample is monitored by an JT-cooled MCT infrared-detector. The lock-in amplifier delivers the amplitude and the phase signal. In order to eliminate any influence from the setup we, usually, normalize the measured phase of the layered sample on the phase signal of a homogeneous infinitely thick layer. In order to fulfil the one-dimensionality of the problem, the spot diameter on the sample's surface for the measurement of the frequency sweeps was adjusted to about $1 \mathrm{~mm}^{2}$, whereas the area observed by the infrared detector is limited to several tens $\mu \mathrm{m}^{2}$. When the samples were scanned, the laser beam was tightly focused on the sample's surface.

\section{Results}

\subsection{Thickness of compound layers on steel}

Carbonitriding belongs to the most important thermochemical hardening treatments of steel. For this case-hardening process the formation of a compound layer at the surface is typical and can be described as a carbonitride layer with a very high surface hardness, high corrosionresistance and very good wear properties. The thickness of the compound layer is usually measured after cutting and grinding of an attended sample. With photothermal radiometry it is possible to measure the thickness non-destructively at each part. Figure 3 shows the calibration 
curve for the determination of the compound layer after carbonitriding on $20 \mathrm{MnCr} 5$ steel. In this way it is possible to measure the layer thickness fast, secure and independent on the sample's geometry and without any necessary sample preparation.

\subsection{Adhesion of galvanically deposited nickel layers on copper}

On galvanically deposited nickel layers we carried out different investigations in order to clarify how a disbonding, in theory described as the thermal contact resistance, influences the photothermal signal and how this compares with the theoretical results. In order to get lack of adhesion the surface of the copper substrate has been passivated in $2 \%$ potassium bichromate solution (DIN EN ISO 8401) before nickel plating. The photothermal phase curves measured at these samples are shown in figure 4. Herein, one can clearly distinguish between good or bad adhesion. The zero-crossing-point of the phase curve of the sample with passivation is moved to lower frequencies compared with the unpassivated one. Assuming that the passivation leads to a thermal contact resistance the measurements are in agreement with the theory. With the destructive tensile test (DIN 50145 and DIN EN ISO 2819) the adhesive force of the passivated layer was measured to be about $10 \mathrm{~N} / \mathrm{mm}^{2}$. For the unpassivated sample the adhesive force cannot be measured, but it can be supposed to be at least a factor of 100 higher. This result shows, that a lack of adhesion can be described by a thermal contact resistance in theory and be defected by the photothermal radiometry. Further experiments will show whether a quantitative determination of adherence with photothermal techniques is possible.

\subsection{Defects in a nickel-palladium layer on copper substrate}

Several photothermal experiments have been performed on $1.2 \mu \mathrm{m}$ thick, galvanically deposited nickel-palladium coatings on $0.3 \mathrm{~mm}$ thick copper alloy sheets. These systems are used for semiconductor devices, e.g., for heat sinks. A few dark spots indicating faults on the surface, a delamination or imperfection at the interface could be found optically. The engineering problem was to characterize these defects by optical and thermal methods and determining if these faults affect adversely the functionality.

In the radiometric investigation the samples have been scanned by stepping motors while the laser beam was tightly focused on the samples' surfaces. The radiometric device remained unchanged, imaging an area of about $25 * 25 \mathrm{~mm}^{2}$. Examplarily for other similar results, figure 5 shows the photothermal phase mapping of a scanned area within $320 \mu \mathrm{m}{ }^{*} 270 \mu \mathrm{m}$. Obviously, some extended 'thermal defects' could be detected. Other lateral scans resulted in imperfections or disbondings, which also could be recognized in the optical images. But, some faults were either seen in the optical or in thermal image. The real extensions of these defects could be determined only by radiometric measurements. E.g., the wide defected area in figure 5 could not be seen in the optical, microscopic image.

Therefore, we carried out frequency sweeps very close and far away from the defected areas. One example is plotted in figure 6 and compared with the theoretically calculated phase contrast curves. On account of the curve shape it may be assumed to have a thermal contact resistance between the nickel-layer and the copper-substrate. For the calculated phase curve we assumed a delamination of the film (air gap) as shown in figure 6 . The fit to the measured values results in a thickness of the air gap of $0.1 \mu \mathrm{m}$. The influence of the delamination or disbonding on the heat propagation is clearly resolved. In particular, these curves indicate a decreased heat-flux at the interface between coating and substrate resulting in an increased reflection and phase shift of the thermal waves.

However, until nowadays we were not able to prove the assumption of a thin air gap. The same effect could also be caused by corrosion or contamination of the copper sheet by other materials. For more detailed determination of the defect type further measurements and comparisons with other, sometimes destructive methods must be done. In these investigations, however, it should be secured that no further defects are induced during the mechanical treatment. 


\section{Conclusions}

The illustrated results give a compressed overview of the applications of photothermal radiometry in non-destructive testing of coated parts. We could show, that a thermal contact resistance can be determined by measuring the phase angles of the radiometric signal as a function of the modulation frequency. Thus, the photothermal technique can be used to detect and determine adhesion defects of various layers on metal or other substrate. By calibration with destructive adherence testing it is possible to estimate the adhesive strength of a decided material combination. The main advantage of this technique is based on the contactless and non-destructive evaluation and the more or less simple measuring setup, easy to insert in industry for an in-line manufacturing control.

However, for a wider acceptance in industry further theoretical modelling is necessary in order to understand photothermal signal generation and verify photothermal measurements. This should be accompanied by a quantitative determination of optical and thermal parameters of organic or galvanic coating materials.

\section{Acknowledgements}

The authors want to thank Dr. A. Zielonka and Mr. U. Heuberger (Forschingsinstitut für Edelmetalle und Metallchemie, Schwäbisch Gmünd) for the preparation of some samples mentioned in the experimental results. The investigations have been financially supported by the Federal State of Baden-Württemberg and an industrial associated project, (Grant No. 44332.62-1 852.18).

\section{REFERENCES}

[1] BUSSE (G.), WALTHER (H.G.): Photothermal nondestructive evaluation of material with thermal waves, in: MANDELIS (A.), Ed: Progress in Photothermal and Photoacoustic Science and Technology, Elsevier, (1992), 207-298

[2] BENNETT (C.A.), PATTY (R.R.): Thermal wave interferometry: a potential application of the photoacoustic effect, Appl. Opt. 21, 1, (1982), 49-54

[3] PATEL (P.M.), ALMOND (D.P.): Thermal wave testing of plasma-sprayed coatings and a comparison of the effects of coating microstructure of the propagation of thermal and ultrasonic waves, J. Mater. Science 20, (1985), 955-966

[4] FRIEDRICH (K.), SEIDEL (U.), WALTHER (H.G.), KARPEN (W.), BUSSE (G.): Proposal for photothermal characterization of boundaries between layer and substrate, Res. Nondestr. Eval. 5, (1993), 31-39

[5] SEIDEL (U.), HAUPT (K.), WALTHER (H.G.): Analysis of the detectability of buried inhomogeneities by means of photothermal microscopy, J.Appl.Phys. 75, 9, (1994), 4396-4401

[6] GOCH (G.), GEERKENS (J.), REICK (M.), SCHMITZ (B.): Analysis of surface layer variations by photothermal means, Journal de Physique IV, C7-4, (1994), 319-322

[7] LAN (T.T.N.), SEIDEL (U.), WALTHER (H.G.), GOCH (G.), SCHMITZ (B.): Experimental results of photothermal microstructural depth profiling, J.Appl. Phys. 78, 6, (1995), 4108-4111

[8] GEERKENS (J.), SCHMITZ (B.), GOCH (G.): Photothermal investigations on advanced ceramics, this conference 


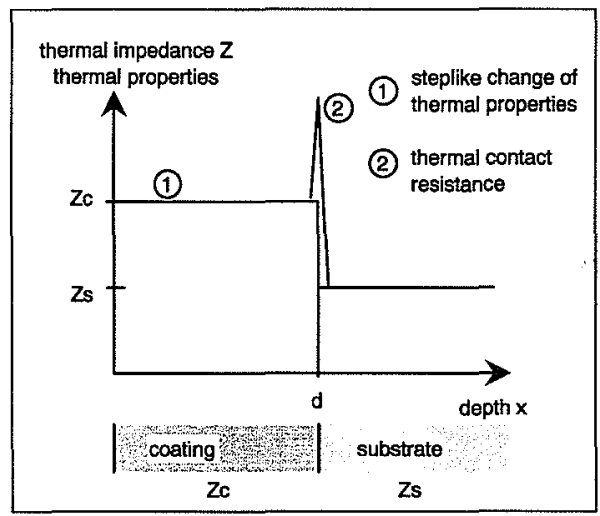

Fig. 1: Variations of the thermal properties at an interface between coating and substrate

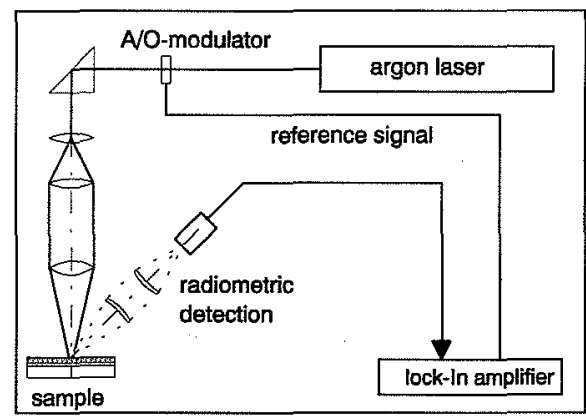

Fig. 2: Experimental setup for photothermal radiometric measurements

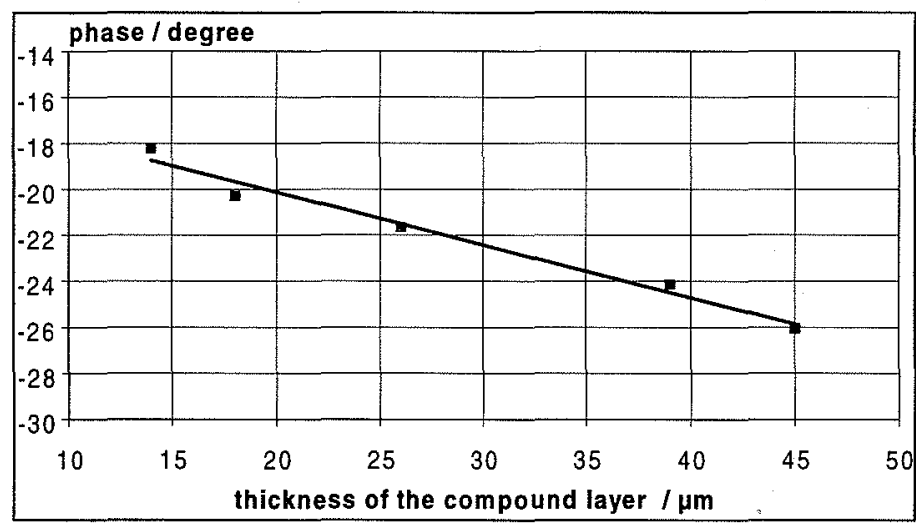

Fig. 3: Calibration curve for the photothermal determination of the compound layer thickness after carbonitriding 
http://dx.doi.org/10.21611/qirt.1996.032

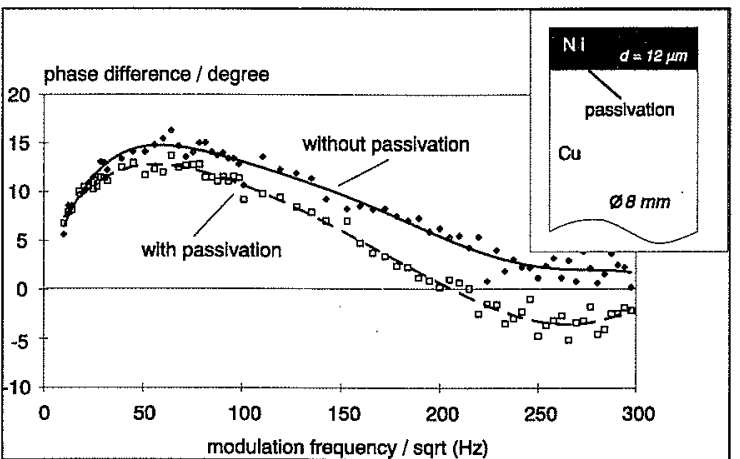

Fig. 4: Photothermal phase curves of the galvanically layered samples with high (wihout passivation) and low adhesive force (with passivation)

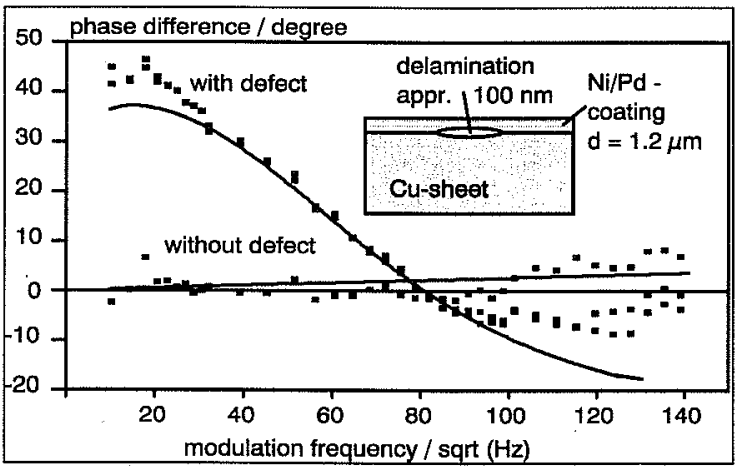

Fig. 6: Sketch of the sample and comparison between theoretical and measured phase behaviour at a thermal contact resistance of a copper sheet with a Ni/Pd coating

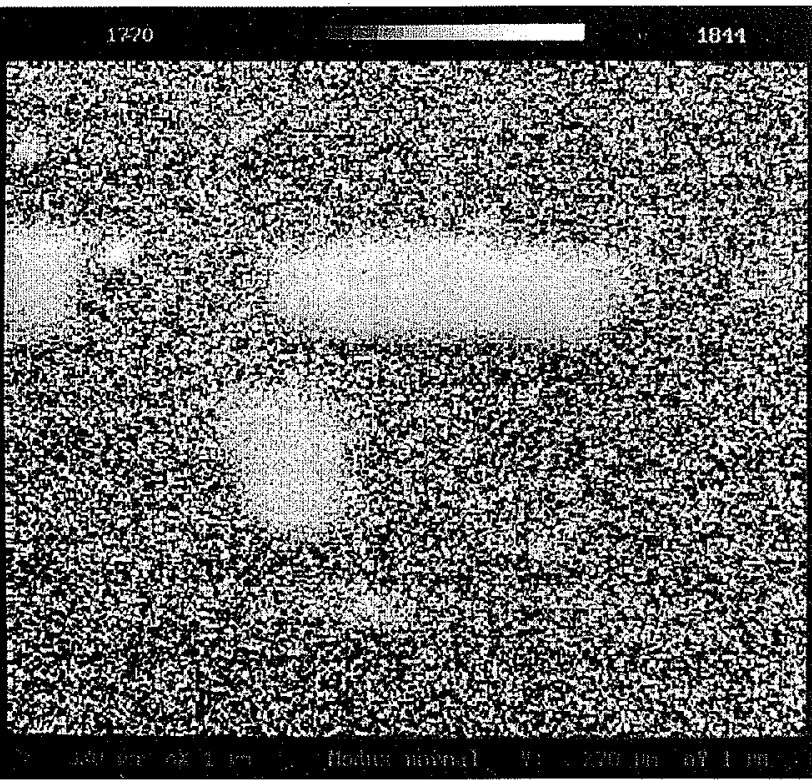

Fig. 5: Photothermal radiometric phase mapping of defects or disbondings between a nickel-palladium protective coating and the copper base material 